\title{
Deep GMRT 150 MHz Observations of the DEEP2 Fields: Searching for High Red-Shift Radio Galaxies Revisited
}

\author{
Susanta K. Bisoi ${ }^{1, *}$, C. H. Ishwara-Chandra ${ }^{2}$, S. K. Sirothia ${ }^{2}$ \\ \& P. Janardhan ${ }^{1}$ \\ ${ }^{1}$ Astronomy \& Astrophysics Division, Physical Research Laboratory, Ahmedabad 380009 , \\ India. \\ ${ }^{2}$ National Centre for Radio Astrophysics, Post Bag No. 3, Ganeshkind, Pune 411 007, India. \\ *e-mail:susanta@prl.res.in
}

\begin{abstract}
High red-shift radio galaxies are best searched at low radio frequencies, due to its steep radio spectra. Here we present preliminary results from our programme to search for high red-shift radio galaxies to $\sim 10$ to 100 times fainter than the known population till date. We have extracted ultra-steep spectrum (USS) samples from deep $150 \mathrm{MHz}$ Giant Meter-wave Radio Telescope (GMRT) observations from one of the three well-studied DEEP2 fields to this effect. From correlating these radio sources with respect to the high-frequency catalogues such as VLA, FIRST and NVSS at $1.4 \mathrm{GHz}$, we find $\sim 100$ steep spectrum (spectral index, $\alpha>1$ ) radio sources, which are good candidates for high red-shift radio galaxies.
\end{abstract}

Key words. Radio source—high red-shift—galaxies.

\section{Introduction}

The high red-shift radio galaxies (HzRGs) have emerged as a unique probe to cosmic evolution containing important information on the physics, and the properties of the early Universe. It had been well established that radio sources with steep radio spectra are more distant than sources with normal spectra. This correlation between $\alpha$ and $z$ has been exploited in the last three decades (Miley \& De Breuck 2008) which yielded about 45 radio galaxies at $z>3$ till date. The median flux density of all of these known HzRGs is $1.3 \mathrm{Jy}$ at $150 \mathrm{MHz}$ which is two orders of magnitude above the detection limit of the GMRT at $150 \mathrm{MHz}$. The GMRT $150 \mathrm{MHz}$ has high angular resolution of $\sim 20^{\prime \prime}$, and better sensitivity of $\sim 1 \mathrm{mJy}$, suggesting that a large number of HzRGs, two orders of magnitude less luminous than all of the known objects, can be detected with GMRT (Ishwara-Chandra et al. 2010). To this effect, we have carried out deep $150 \mathrm{MHz}$ observations of three of the four DEEP2 (Deep Extragalactic Evolutionary Probe 2) fields, which has optical spectra of $\sim 50,000$ objects. 

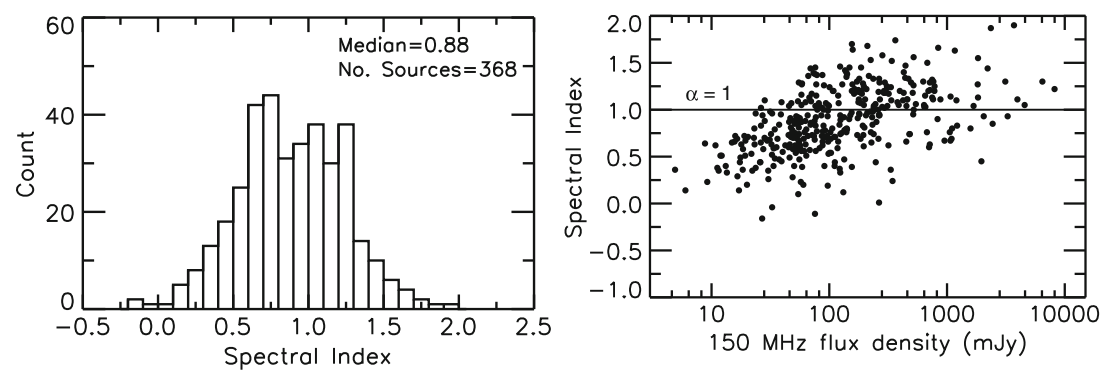

Figure 1. Histogram of spectral index of sources (left) and spectral index distribution of sources as a function of flux density at GMRT $150 \mathrm{MHz}$ (right).

\section{Result and discussion}

One of the deep fields, centered on $2330+0000$, from DEEP2 was analysed using AIPS ++ (Sirothia et al. 2009), and the final image obtained has an rms noise $\sim 1 \mathrm{mJy} / \mathrm{beam}$ with a resolution of $20^{\prime \prime}$. The $150 \mathrm{MHz}$ source catalog of this field contains $\sim 400$ radio sources to $20 \%$ peak primary beam response with flux density limit of $\sim 6 \mathrm{mJy}$ while the median flux density is $\sim 100 \mathrm{mJy}$. The spectral index is estimated by comparison of $150 \mathrm{MHz}$ flux density with that of NVSS and FIRST at $1.4 \mathrm{GHz}$. A total of 368 sources from GMRT $150 \mathrm{MHz}$ catalog has counterparts found at FIRST and/or NVSS. Figure 1 shows the spectral index distribution of sources as a function of $150 \mathrm{MHz}$ flux density. Above a cutoff of $\alpha=1$, there are about 100 steep spectrum sources. The FIRST position of these sources will be used to search SDSS and DEEP2 optical catalogs to eliminate nearby radio sources, and the unidentified radio sources will be the potential candidates for HzRGs.

\section{References}

Ishwara-Chandra, C. H., Sirothia, S. K., Wadadekar, Y., Pal, S., Windhorst, R. 2010, Mon. Not. R. Astron. Soc., 405, 436.

Miley, G., De Breuck, C. 2008, Astron. Astrophys. Rev., 15, 67.

Sirothia, S. K., Saikia, D. J., Ishwara-Chandra, C. H., Kantharia, N. G. 2009, Mon. Not. R. Astron. Soc., 392, 1403. 